\title{
On the Chabauty space of locally compact abelian groups
}

\author{
YVES CORNULIER
}

\begin{abstract}
This paper contains several results about the Chabauty space of a general locally compact abelian group. Notably, we determine its topological dimension, we characterize when it is totally disconnected or connected; we characterize isolated points.
\end{abstract}

22B05; 20E15, 43A25, 54D05, 54F45

\section{Introduction}

Let $X$ be a locally compact Hausdorff space. The set $\mathcal{F}(X)$ of closed subsets can be endowed with the Chabauty topology, which makes it a compact Hausdorff space. For this topology, a net $\left(Y_{i}\right)$ converges to $Y$ if and only if $\left(Y_{i} \cup\{\infty\}\right)$ converges to $Y \cup\{\infty\}$ in the Hausdorff topology of the one-point compactification of $X$. When $X$ is second countable, $\mathcal{F}(X)$ is metrizable. See details in Section 2.1. If $G$ is a locally compact group, the set $\mathcal{S}(G)$ of closed subgroups of $G$ is closed in $\mathcal{F}(G)$ and therefore is compact Hausdorff as well.

Introduced by Chabauty in [7], the Chabauty topology has been studied by Hubbard and Pourezza [24], Protasov [25], Protasov and Tsybenko [27; 26], Fisher and Gartside [13; 14], Bridson, de la Harpe and Kleptsyn [6], Kloeckner [20], Haettel [16], and more specifically for discrete groups by Grigorchuk [15], Champetier [8], and by the author together with Guyot and Pitsch $[9 ; 10]$. The fine study of $\mathcal{S}(G)$ is subtle even for apparently simple examples. While it is readily seen that $\mathcal{S}(\mathbb{R})$ is homeomorphic to a segment, a tricky argument due to Hubbard and Pourezza [24] shows that $\mathcal{S}\left(\mathbb{R}^{2}\right)$ is homeomorphic to the 4-sphere. For $n \geq 3, \mathcal{S}\left(\mathbb{R}^{n}\right)$ is known to be singular (that is, not a topological manifold, even with boundary) but Kloeckner [20] showed that $\mathcal{S}\left(\mathbb{R}^{n}\right)$ has a natural "stratification" which in particular makes it a simply connected and locally contractible space; however $\mathcal{S}\left(\mathbb{R}^{n}\right)$ has not yet unveiled all its mysteries and for instance it is still unknown whether it can be triangulated. Besides, Haettel [16] gave a full description of the space $\mathcal{S}(\mathbb{R} \times \mathbb{Z})$, showing in particular that it is path-connected but not locally connected, and has uncountable fundamental group.

Our first result, which underlies the proof of all others, is the continuity of the orthogonal map in Pontryagin duality. Let $G$ be an LCA-group (by this acronym we mean a locally 
compact abelian group). Under the topology of uniform convergence on compact subsets, the abelian group $G^{\vee}=\operatorname{Hom}(G, \mathbb{R} / \mathbb{Z})$ is locally compact, and the main result in Pontryagin duality is that the natural homomorphism $G \rightarrow\left(G^{\vee}\right)^{\vee}$ is a topological group isomorphism. In Bourbaki [3, Chapitre II.2], Pontryagin duality is used to deduce fundamental results in the structure theory on LCA-groups. One of the results $[3$, Chapitre II.1, no. 7] is that the orthogonal map

$$
\begin{aligned}
\mathcal{S}(G) & \rightarrow \mathcal{S}\left(G^{\vee}\right) \\
H & \mapsto H^{\curlywedge}=\{\phi \in \operatorname{Hom}(G, \mathbb{R} / \mathbb{Z}): \phi(H)=0\}
\end{aligned}
$$

is a bijection.

Theorem 1.1 Let $G$ be an LCA-group. Then the orthogonal map $\mathcal{S}(G) \rightarrow \mathcal{S}\left(G^{\vee}\right)$, mapping $H \subset G$ to its orthogonal $H^{\wedge}$, is a homeomorphism.

We refer to this result as Pontryagin-Chabauty duality, and we develop consequences on the general structure of the space $\mathcal{S}(G)$. The next theorem deals with the topological (or covering) dimension, which is a (possibly infinite) integer number $\operatorname{tdim}(X)$ associated to any topological space $X$, invariant under homeomorphism, and for which $\operatorname{tdim}\left(\mathbb{R}^{n}\right)=n$ for all $n$. See Section 2.2 for details.

Theorem 1.2 (Theorem 4.7) If $G$ is any LCA-group, then the topological (covering) dimension of $\mathcal{S}(G)$ is given by

$$
\operatorname{tdim}(\mathcal{S}(G))=\operatorname{tdim}(G) \operatorname{tdim}\left(G^{\vee}\right),
$$

where $0 \infty=\infty 0=0$. In particular, if $G=\mathbb{R}^{d} \times \mathbb{Z}^{\ell} \times \mathbb{R} / \mathbb{Z}^{m}$ then

$$
\operatorname{tdim}(\mathcal{S}(G))=(d+\ell)(d+m) .
$$

Several characterizations of $\operatorname{tdim}(G)$ and $\operatorname{tdim}\left(G^{\vee}\right)$ are recalled in Section 2.8. The above result Theorem 1.2 is based on the special case

$$
\operatorname{tdim}\left(\mathcal{S}\left(\mathbb{R}^{d}\right)\right)=d^{2},
$$

which holds because its has a decomposition into finitely many orbits under the action of $\mathrm{GL}_{d}(\mathbb{R})$, each of which is a manifold of dimension at most $d^{2}$, and one of which is exactly $d^{2}$-dimensional, namely the set of lattices (see Proposition 4.2).

If $G$ is an LCA-group, from classical theory it can be written as $\mathbb{R}^{k} \times M$ so that $M$ has a compact open subgroup; the finite number $R(G)=k$ is uniquely defined (see Section 2.6). The following results bring out a dichotomy between the case $R(G)=0$ (that is, $G$ is compact-by-discrete) and the case $R(G) \geq 1$. 
Theorem 1.3 (Section 8) Let $G$ be an LCA-group with $R(G) \geq 1$. Then $\mathcal{S}(G)$ is connected. If moreover $G$ is a Lie group and is compactly generated (that is, $G / G_{0}$ is finitely generated, where $G_{0}$ is the unit component), then $\mathcal{S}(G)$ is path-connected.

The connectedness statement was obtained by Protasov and Tsybenko [26] (see the discussion in Section 8). Nevertheless, Proposition 8.5 exhibits countable discrete abelian groups $A$ such that $\mathcal{S}(\mathbb{R} \times A)$ is not path-connected.

Example 1.4 By Theorems 1.2 and 1.3, if $G$ is an LCA-group, then $\mathcal{S}(G)$ is both connected and one-dimensional if and only if $G \simeq \mathbb{R} \times H$, where $H$ is profinite-by(discrete torsion). In many cases, like $\mathbb{R} \times \mathbb{Q}_{p}$, it also follows from Theorem 8.4 that $\mathcal{S}(G)$ is path-connected. It would be interesting to have a closer look into $\mathcal{S}(G)$ for those examples.

If $X$ is compact Hausdorff space, we define $\pi_{0}(X)$ as the quotient of $X$ by its partition by connected components. By Bourbaki [4, II.4.4, Proposition 7], $\pi_{0}(X)$ is compact, Hausdorff and totally disconnected. Section 7 studies LCA-groups $G$ with $R(G)=0$ and more precisely the connected components of $\mathcal{S}(G)$. For such groups, it is readily observed that $\mathcal{S}(G)$ is not connected unless $G=\{0\}$ (this is for instance a particular case of a result in [26] pertaining to non-abelian locally compact groups). Our results yield in particular

Theorem 1.5 Let $G$ be an LCA-group with $R(G)=0$. Then

- $\pi_{0}(\mathcal{S}(G))$ is infinite if and only if $G$ is infinite;

- every connected component of $\mathcal{S}(G)$ is homeomorphic to a compact group; if $G$ is a compactly generated Lie group, these components are tori.

- $\mathcal{S}(G)$ is totally disconnected if and only if $G$ is either totally disconnected or elliptic (that is, it is the union of its compact subgroups).

Also, Theorem 7.9 gives a structure result for LCA-groups for which $\mathcal{S}(G)$ has countably many connected components. We extract from it

Theorem 1.6 Let $G$ be an LCA-group which is neither discrete nor compact. Then $\mathcal{S}(G)$ has (at most) countably many connected components if and only if one of the following three conditions holds

- $G=\mathbb{Q}_{\ell} \times \mathbb{Z}_{m} \times \mathbb{C}_{n} \infty \times F$, where $F$ is finite, $\ell, m, n \geq 1, \ell$ is prime with $m n$;

- $G=K \times D \times F$ with $D$ torsion-free discrete, $K$ compact connected, $F$ finite, and $\mathcal{S}(D)$ and $\mathcal{S}(K)$ are countable; 
- $R(G) \geq 1$.

Note that in the first case $G$ is both elliptic and totally disconnected so $\mathcal{S}(G)$ is countable itself, while in the second case $\mathcal{S}(G)$ is not totally disconnected by Theorem 1.5. Note that the case when $G$ is discrete (characterization of abelian groups with countably many subgroups) is done by Boyer [5] (see Proposition 7.8) and the compact case follows by Pontryagin duality. Note that the proof of Theorem 1.6 involves an intrinsic characterization of finite direct products of $p$-adic groups $\mathbb{Q}_{p}$ (see Lemma 7.10).

The next result concerns isolated points. When $G$ is a discrete abelian group, a necessary and sufficient condition for a subgroup $H$ to be isolated in $\mathcal{S}(G)$ is that $H$ is isolated in $\mathcal{S}(H)$ and $\{1\}$ is isolated in $\mathcal{S}(G / H)$ (see for instance the paper [10] by the author, Guyot and Pitsch). However when $G$ is not assumed discrete, this condition obviously remains necessary but is not sufficient: for instance $\mathbb{Z}$ is not isolated in $\mathcal{S}(\mathbb{R})$. The characterization of isolated points goes as follows (we refer to Section 2.4 for the definition of Artinian and adic groups)

Theorem 1.7 Let $G$ be an LCA-group and $H$ a closed subgroup. Then $H$ is an isolated point in $\mathcal{S}(G)$ if and only if we are in one of the two (dual to each other) cases

(1) $H \simeq A \times P, G / H \simeq D, A$ finitely generated abelian, $P$ adic, $D$ Artinian (so $G$ is totally disconnected);

(2) $H \simeq P, G / H \simeq T \times D, P$ adic, $T$ torus, $D$ Artinian (so $G$ is elliptic).

In [10], the study was pursued to a complete description of the homeomorphism type of $\mathcal{S}(G)$, when $G$ is a countable discrete abelian group. It would be interesting to generalize this to second countable, totally disconnected LCA-groups. Among those groups, those for which this question is nontrivial have a very special form: they have to lie in an exact sequence

$$
0 \rightarrow A \times P \rightarrow G \rightarrow D \rightarrow 0
$$

with $A, P, D$ as in Theorem 1.7(1), since if $G$ is not of this form, by the same theorem, $\mathcal{S}(G)$ has no isolated point and therefore is a Cantor space.

Acknowledgements I thank P de la Harpe, B Kloeckner and R Tessera for useful comments. I also thank the referee, notably for mentioning the book by Engelking [12] which led to a substantial simplification of computation of the dimension of $\mathcal{S}\left(\mathbb{R}^{d}\right)$, as well as for various remarks improving the presentation of the paper. 


\section{Preliminaries}

This section recalls definitions and basic results used throughout the paper.

\subsection{The Chabauty topology}

Recall that if $X$ is a locally compact space, we denote by $\mathcal{F}(X)$ the set of closed subsets of $X$. This set has several natural topologies. The one of interest for us is called the Chabauty topology (or compact topology), and is compact Hausdorff. It appears in an exercise by Bourbaki [2, Chapitre 8, Section 5] and was reintroduced by Narens [22] in the context and language of non-standard analysis and its description in standard terms was then provided by Wattenberg [29]. Given a compact subset $K \subset X$ and open subsets $U_{1}, \ldots, U_{k} \subset X$, define

$$
\Omega\left(K ; U_{1}, \ldots, U_{k}\right)=\left\{F \in \mathcal{F}(X): F \cap K=\varnothing \text {; for all } i, F \cap U_{i} \neq \varnothing\right\} ;
$$

these set form the basis of the Chabauty topology on $\mathcal{F}(X)$.

The reader can prove as a simple exercise the following characterization of converging nets in $\mathcal{F}(X)$.

Lemma 2.1 For a net $\left(F_{i}\right)$ of closed subsets of $X$ and $F \in \mathcal{F}(X)$, we have equivalences

- $F_{i} \rightarrow F$ in the Chabauty topology;

- for every compact subset $K$ and any open subsets $U_{1}, \ldots, U_{k}$ with $F \cap K=\varnothing$ and $F \cap U_{j} \neq \varnothing$ for each $j$, we have eventually $F_{i} \cap K=\varnothing$ and $F_{i} \cap U_{j} \neq \varnothing$ for each $j$;

- for every $x \in F$ and every neighbourhood $V$ of $x$, we eventually have $F_{i} \cap V \neq$ $\varnothing$, and for every $x \in X-F$ there exists a neighbourhood $V$ of $x$ such that eventually $F_{i} \cap V=\varnothing$.

From the latter characterization it is straightforward that if $X=G$ is a locally compact group, then $\mathcal{S}(G)$ is closed in $\mathcal{F}(X)$; in this specific case and under countability assumptions, this topology was introduced by Chabauty [7].

\subsection{Dimension of topological spaces}

We recall briefly several notions of dimension. For details see Hurewicz and Wallman [17]. Let $X$ be a topological space. If $\mathcal{U}=\left(U_{i}\right)_{i \in I}$ is an open covering of $X$, define its degree as

$$
\operatorname{deg}(\mathcal{U})=\sup \left\{n: \text { there exists } J \subset I, \# J=n, \bigcap_{i \in J} U_{i} \neq \varnothing\right\},
$$


and the topological dimension (or covering dimension) $\operatorname{tdim}(X)$ of $X$ is defined as the smallest $n$ such that every open covering of $X$ can be refined to an open covering with degree $\leq n+1$ (by convention $\operatorname{tdim}(\varnothing)=-1$ ). This dimension is well-behaved in many respects, for instance $\operatorname{tdim}\left(\mathbb{R}^{n}\right)=n$.

The inductive dimension indim $(X)$ of a topological space $X$ is defined inductively as follows: $\operatorname{indim}(X)=-1$ if and only if $X=\varnothing$; otherwise indim $(X) \leq n$ if and only if every $x \in X$ has a basis of closed neighbourhoods $\left(V_{i}\right)$ such that for each $i$, the boundary of $V_{i}$ in $X$ has inductive dimension $\leq n-1$.

These dimensions are related. Let us state, for later reference

\section{Proposition 2.2}

(1) By a theorem of Urysohn (see Hurewicz and Wallman [17]), if $X$ is a separable metrizable space, then $\operatorname{indim}(X)=\operatorname{tdim}(X)$. This applies in particular to $X=\mathcal{S}(G)$ when $G$ is a second countable locally compact group.

(2) By a theorem of Pasynkov [23], if $G$ is a locally compact group, then $\operatorname{indim}(G)=$ $\operatorname{tdim}(G)$.

(3) A theorem of Aleksandrov (see Isbell [18]) states that if $X$ is any compact Hausdorff space then $\operatorname{tdim}(X) \leq \operatorname{indim}(X)$; this is an equality when $X$ is metrizable by (1) but not for general compact Hausdorff spaces (see Vopěnka [28]).

(4) The topological dimension of an inverse limit of Hausdorff compact spaces of topological dimension $\leq k$ is also $\leq k$ (see Katuta [19]).

\subsection{Scattered spaces}

A topological space is scattered if every non-empty subset has an isolated point, or equivalently if every non-empty closed subset has an isolated point. Every countable compact Hausdorff space is scattered, as a consequence of the Baire Category Theorem.

\subsection{Subclasses of groups}

Recall that, for $n \geq 2$, the Prüfer group $\mathbb{C}_{n} \infty$ is defined as the inductive limit of the groups $\mathbb{C}_{n^{k}}=\mathbb{Z} / n^{k} \mathbb{Z}$; in particular $\mathbb{C}_{n^{\infty}} \simeq \mathbb{Z}[1 / n] / \mathbb{Z}$ and is the direct product of $\mathbb{C}_{p} \infty$ where $p$ ranges over distinct prime divisors of $n$. Similarly, the ring $\mathbb{Z}_{n}$ of $n$-adic numbers is defined as the projective limit of the groups $\mathbb{Z} / n^{k} \mathbb{Z}$ and is the product of $\mathbb{Z}_{p}$ where $p$ ranges over distinct prime divisors of $n$. Also, $\mathbb{Q}_{n}$ denotes the product of $p$-adic fields $\mathbb{Q}_{p}$ when $p$ ranges over distinct divisors of $n$, and we call a group local if it is isomorphic to a finite direct product of $\mathbb{Q}_{p}$ (distinct or not). 
Recall that a discrete abelian group is artinian if it satisfies the descending condition on subgroups (no infinite decreasing chain), or equivalently is a finite direct product of Prüfer groups and finite groups. We say here that an LCA-group is adic if it is a finite direct product of finite groups and groups of the form $\mathbb{Z}_{n}$ for some (non-fixed) $n$.

We call torus a group of the form $\mathbb{R}^{k} / \mathbb{Z}^{k}$.

An abelian $A$ (without topology) is divisible if the map $g \mapsto n g$ is surjective for all $n \geq 1$. This is equivalent to the fact that whenever $A$ is a subgroup of an abelian group $B$, it has a direct factor in $B$. An LCA-group is divisible if the underlying discrete group is divisible. A connected LCA-group is always divisible; conversely a compact divisible LCA-group is connected.

An LCA-group $G$ is elliptic if any element belongs to a compact subgroup; it actually implies that any compact subset belongs to a compact subgroup. It is the same as compact-by-(discrete torsion). The largest elliptic subgroup of an LCA-group is a closed subgroup, denoted by $E_{G}$.

If $G$ is a compact group and $I$ an abstract set, the full direct product $G^{I}$ is still a compact group. If $G$ is a discrete group, the restricted direct product $G^{(I)}$ is defined as the set of finitely supported functions $I \rightarrow G$, and is endowed with the discrete topology.

A compact group is always a filtering projective limit of compact Lie groups. (We only use this fact in the abelian case, where it is a plain consequence of Pontryagin duality.)

\subsection{On Pontryagin duality}

The following tables shows various groups or classes of LCA-groups, in correspondence by Pontryagin duality. See Bourbaki [3, Chapitre II.2] for details.

\begin{tabular}{|c|c|}
\hline$G$ & $G^{\vee}$ \\
\hline $\mathbb{R}$ & $\mathbb{R}$ \\
\hline $\mathbb{Q}_{p}$ & $\mathbb{Q}_{p}$ \\
\hline $\mathbb{Z} / n \mathbb{Z}$ & $\mathbb{Z} / n \mathbb{Z}$ \\
\hline $\mathbb{Z}$ & $\mathbb{R} / \mathbb{Z}$ \\
\hline $\mathbb{Z}_{p}$ & $\mathbb{C}_{p} \infty$ \\
\hline
\end{tabular}




\begin{tabular}{|c|c|}
\hline class $\mathcal{C}$ & $\mathcal{C}^{\vee}$ \\
\hline discrete & compact \\
\hline Lie & compactly generated \\
\hline totally disconnected & elliptic \\
\hline discrete torsion-free & connected compact \\
\hline discrete torsion & profinite \\
\hline discrete artinian & adic \\
\hline discrete finitely generated & torus-by-finite \\
\hline
\end{tabular}

\subsection{The invariant $R$}

Let $G$ be an LCA-group. Define

$$
R(G)=\sup \left\{k: \mathbb{R}^{k} \text { is isomorphic to a direct factor of } G\right\} .
$$

The following result is contained in Bourbaki [3, II.2, Proposition 3(i)]

Proposition 2.3 Every LCA-group $G$ is isomorphic to the direct product of $\mathbb{R}^{k}$ and a compact-by-discrete group.

If $G$ is a compactly generated abelian Lie group, then it isomorphic to $\mathbb{R}^{k} \times \mathbb{Z}^{\ell} \times$ $(\mathbb{R} / \mathbb{Z})^{m} \times F$ with $F$ finite and $k, \ell, m$ non-negative integers.

From this, we immediately derive the following results.

\section{Lemma 2.4}

- If $G_{1}$ is an open subgroup of $G_{2}$, then $R\left(G_{1}\right)=R\left(G_{2}\right)$.

- If $K$ is a compact subgroup of $G$ then $R(G / K)=R(G)$.

- For every LCA-group, $R(G)<\infty$.

- We have $R(G)=0$ if and only if $G$ is compact-by-discrete.

- $R(G)=R\left(G^{\vee}\right)$.

\subsection{On connected groups}

Lemma 2.5 If a connected compact LCA-group $G$ is non-trivial, then it contains a nontrivial path.

Proof As $G^{\vee}$ is a non-trivial discrete torsion-free abelian group (see Section 2.5), we have $\operatorname{Hom}\left(G^{\vee}, \mathbb{R}\right) \neq 0$. By Pontryagin duality, $\operatorname{Hom}(\mathbb{R}, G)$ is non-trivial as well. In particular, $G$ contains non-trivial paths. 
Lemma 2.6 Let $D$ be a torsion-free discrete abelian group and $Q$ a connected compact abelian group. If both $D$ and $Q$ are non-zero, then $\operatorname{Hom}(D, Q)$ is a non-trivial compact connected group.

Proof We have a non-zero element $f$ in $\operatorname{Hom}(D, \mathbb{R})$ and by Pontryagin duality, we can find a non-zero $g$ in $\operatorname{Hom}(\mathbb{R}, Q)$ as well. By composing $g$ by $\lambda f$ for suitable $\lambda>0$, we get a non-trivial element in $\operatorname{Hom}(D, Q)$.

As $D$ is a direct limit of groups of the form $\mathbb{Z}^{k}, \operatorname{Hom}(D, Q)$ is a projective limit of groups of the form $\operatorname{Hom}\left(\mathbb{Z}^{k}, Q\right)=Q^{k}$ which are compact and connected. Therefore $\operatorname{Hom}(D, Q)$ is compact and connected as well.

\subsection{Dimension of LCA-groups}

We deal here with the topological dimension because we need to use Proposition 2.2(4), but by (2) of the same proposition, it coincides for locally compact groups with the inductive dimension. The following lemma is a particular case of Dixmier [11, Theorem 5]. Recall that all homomorphisms are assumed to be continuous.

Lemma 2.7 If $G$ is an LCA-group, $H$ a closed subgroup and $f: H \rightarrow \mathbb{R}$ a homomorphism, then $f$ can be extended to a homomorphism $G \rightarrow \mathbb{R}$.

Proof Let $K$ be a compact subgroup of $G$ such that $G / K$ is a Lie group whose unit component is isomorphic to $\mathbb{R}^{k}$. Working in $G / K$ if necessary, we can suppose $K=0$. Let $H_{1}$ be the inverse image in $G$ of the torsion in $G / H$. Then $f$ has a unique extension to $H_{1}$; in restriction to $H_{1} \cap G_{0}$ this extension is continuous as it essentially consists in extending a homomorphism from $\mathbb{R}^{\ell} \times \mathbb{Z}^{m}$ to $\mathbb{R}^{\ell+m}$. Now there exists a direct factor of $H_{1} \cap G_{0}$ in $G_{0}$, so $f$ extends to $H+G_{0}$, and finally by injectivity of the $\mathbb{Z}$-module $\mathbb{R}$, we can extend $f$ to all of $G$.

Lemma 2.8 The following (possibly infinite) numbers are equal:

- The supremum of $k$ such that there exists a homomorphism $G \rightarrow \mathbb{R}^{k}$ such that the closure of the image is cocompact

- The supremum of $k$ such that $\mathbb{Z}^{k}$ embeds discretely into $G$.

Proof Suppose that $G \rightarrow \mathbb{R}^{k}$ is a homomorphism whose image has cocompact closure. Then the image contains a basis, hence a lattice, which lifts to a discrete subgroup of $G$ isomorphic to $\mathbb{Z}^{k}$.

Suppose that $\mathbb{Z}^{k}$ embeds discretely into $G$. Consider the embedding of $\mathbb{Z}^{k}$ in $\mathbb{R}^{k}$ as a lattice. By Lemma 2.7, this can be extended to a homomorphism $G \rightarrow \mathbb{R}^{k}$ whose image has cocompact closure. 
Lemma 2.9 The following (possibly infinite) numbers are equal

- The topological dimension $\operatorname{tdim}(G)$;

- The supremum of $k$ such that there exists a homomorphism $\mathbb{R}^{k} \rightarrow G$ with discrete kernel;

- The supremum of $k$ such that $(\mathbb{R} / \mathbb{Z})^{k}$ is a quotient of $G$.

Proof Denote by $a, b, c$ the corresponding numbers. By Lemma 2.8 and Pontryagin duality, $b=c$. Clearly $a \geq b$.

Let us prove $a \leq c$. The three numbers are invariant if we replace $G$ by an open subgroup, so we suppose $G / G_{0}$ compact. Therefore we can write $G=\mathbb{R}^{\ell} \times K$ with $K$ compact, and we write $K$ as a filtering projective limit of compact Lie groups $K_{i}$. If $c=c(G)=k+\ell<\infty$, then $c\left(K_{i}\right) \leq k$ for all $i$, so $K_{i}$ is a compact Lie group of dimension at most $k$, hence of topological dimension at most $k$. By Proposition 2.2(4), $K$ has dimension at most $k$, so $G$ has dimension at most $k+\ell$.

By Pontryagin duality again, we get

Corollary 2.10 The numbers in Lemma 2.8 are also equal to the topological dimension of $G^{\vee}$.

We will also need the following lemma.

Lemma 2.11 Let $G$ be an LCA-group and $k=R(G)$. There exist abstract sets $I, J$, a compact subgroup $K$ in $G$ and an open subgroup $H$ containing $K$, such that

- $H / K$ is isomorphic to $\mathbb{Z}^{(I)} \times(\mathbb{R} / \mathbb{Z})^{J} \times \mathbb{R}^{k}$,

- $\operatorname{dim}(G)=\operatorname{dim}(H / K)=k+\# J$, and

- $\operatorname{dim}\left(G^{\vee}\right)=\operatorname{dim}\left((H / K)^{\vee}\right)=k+\# I$.

(Here the cardinal of an infinite set is just $\infty$.)

Proof In view of Proposition 2.3, we can suppose $G$ has a compact open subgroup $M$. Consider a maximal free subgroup $H / M$ in the discrete group $G / M$. Obviously $\operatorname{dim}(G)=\operatorname{dim}(H)$, and since $G / H$ is torsion, it follows from Corollary 2.10 that $\operatorname{dim}\left(G^{\vee}\right)=\operatorname{dim}\left(H^{\vee}\right)$. Apply this to find, by duality, a closed group $K$ of $M$ such that $(M / K)^{\vee}$ is maximal free abelian, so $\operatorname{dim}(H)=\operatorname{dim}(H / K)$ and $\operatorname{dim}\left(H^{\vee}\right)=$ $\operatorname{dim}\left((H / K)^{\vee}\right)$. Now $M / K$ is connected, hence divisible, and open in $H / K$, so has a direct factor. So $H / K$ is isomorphic to $\mathbb{Z}^{(I)} \times(\mathbb{R} / \mathbb{Z})^{J}$ for some sets $I, J$. 


\section{Pontryagin-Chabauty duality}

Theorem 1.1 is stated by Protasov [25], and the short and elementary proof given therein consists in an elegant reduction to the case of a Euclidean space, but the latter case is only considered there as "easily verified". Also, continuity of the orthogonal map in $\mathcal{S}\left(\mathbb{R}^{d}\right)$ is asserted by Kloeckner [20, Section 2.4]. We here give a detailed proof of this not-so-obvious fact.

Theorem 3.1 Let $V$ be a finite-dimensional real vector space. The orthogonal map $\mathcal{S}(V) \rightarrow \mathcal{S}\left(V^{\vee}\right)$, is a homeomorphism.

We need a few preliminary results.

Lemma 3.2 Let $W$ be a closed subgroup of $V$ and $\Gamma$ a lattice in $W$. Let $\left(W_{n}\right)$ be a sequence of closed subgroups of $V$ such that $W_{n} \rightarrow W$. Then there exists $A_{n} \in \mathrm{GL}(V)$ with $A_{n} \rightarrow 1$ and $\Gamma \subset A_{n} W_{n}$.

Proof We can suppose $V=\mathbb{R}^{d}$ with canonical basis $\left(e_{1}, \ldots\right), W=\mathbb{R}^{k} \times \mathbb{Z}^{\ell} \times$ $\{0\}^{d-k-\ell}, \Gamma=\mathbb{Z}^{k+\ell} \times\{0\}^{d-k-\ell}$.

We can pick, for $i \leq k+\ell, e_{i}^{(n)} \in W_{n}$ so that $e_{i}^{(n)} \rightarrow e_{i}$ for all $i$. Consider the linear map $B_{n}$ mapping each $e_{i}$ to $e_{i}^{(n)}$ (agree $e_{i}^{(n)}=e_{i}$ for $i>k+\ell$ ). Then $B_{n} \rightarrow 1$, so eventually $B_{n}$ has an inverse $A_{n}$, and $\Gamma \subset A_{n} W_{n}$.

When $V$ is a finite-dimensional real vector space, there is a canonical identification between the (ordinary) dual $V^{*}$ and the Pontryagin dual $V^{\vee}$, given by composition of a linear mapping $V \rightarrow \mathbb{R}$ by the natural projection $\mathbb{R} \rightarrow \mathbb{R} / \mathbb{Z}$. Then if $W$ a closed subgroup of $V, W^{\curlywedge}$ corresponds under this identification to

$$
\left\{L \in V^{*}: \text { for all } v \in W,\langle v, L\rangle \in \mathbb{Z}\right\},
$$

which is called, when $W$ is a lattice, the dual lattice. If $A \in \mathrm{GL}(V)$, then it is immediate that $(A W)^{\curlywedge}=\left(A^{t}\right)^{-1} W^{\curlywedge}$, where $A^{t} \in \mathrm{GL}\left(V^{*}\right)$ is the adjoint map of $A$.

Lemma 3.3 Let $W$ be a closed subgroup of $V$ and $\Gamma$ a lattice in $W$. Let $W_{n}$ be a sequence of closed subgroups of $V$ such that $W_{n} \rightarrow W$. Suppose that $W_{n}$ eventually contains $\Gamma$. Then $W_{n} \cap W^{0} \rightarrow W^{0}$ (the unit component of $W$ ).

Proof Let $w \in W^{0}$. Then there exists a sequence $w_{n} \in W_{n}$ with $w_{n} \rightarrow w$. Set $R=d\left(W^{0}, W-W^{0}\right)$. We can suppose $d\left(w_{n}, W^{0}\right) \leq 2 R / 3$ for all $n$. If $w_{n} \notin W^{0}$ for infinitely many $n$ 's, we can find a positive integer $m_{n}$ such that $d\left(m_{n} w_{n}, W^{0}\right)=$ 
$m_{n} d\left(w_{n}, W_{0}\right) \in[R / 3,2 R / 3]$. Now we can find $\gamma_{n} \in \Gamma$ such that the sequence $\left(m_{n} w_{n}-\gamma_{n}\right)$ is bounded. By assumption it belongs to $W_{n}$, and $d\left(m_{n} w_{n}-\gamma_{n}, W^{0}\right) \in$ $[R / 3,2 R / 3]$. So it has a cluster value $x$ with $d\left(x, W^{0}\right) \in[R / 3,2 R / 3]$, so $x \notin W$, but $W_{n} \rightarrow W$ forces $x \in W$, a contradiction, that is, $w_{n} \in W^{0}$ for large $n$.

Proposition 3.4 There exists a constant $C_{d}$ with the following property. Whenever $\Gamma$ is a discrete subgroup of $\mathbb{R}^{d}$ with shortest vector of length $\geq R$, then $\Gamma^{\vee}$ is $\varepsilon$-dense, with $\varepsilon=C_{d} / R$.

Proof We can embed $\Gamma$ into a lattice having the same shortest vector. This reduces to the classical case of lattices, due to Mahler [21] (an elementary approach provides $C_{d}$ with an exponential upper bound with respect to $d$; nevertheless a linear bound on $C_{d}$ can be obtained, see Banaszczyk [1]).

Proof of Theorem 3.1 We identify $V=\mathbb{R}^{d}$. Suppose $H_{n}$ tends to $H=\mathbb{R}^{k} \times$ $\mathbb{Z}^{\ell} \times\{0\}^{m}(k+\ell+m=d)$ and let us prove that $H_{n}^{\vee}$ tends to $\{0\}^{k} \times \mathbb{Z}^{\ell} \times \mathbb{R}^{m}$. Taking subsequences if necessary, we can suppose these sequences converge. Clearly the limit $W$ of $H_{n}^{\vee}$ is " $\mathbb{R} / \mathbb{Z}$-orthogonal" to $H$ and therefore $W$ is contained in $\{0\}^{k} \times \mathbb{Z}^{\ell} \times \mathbb{R}^{m}$.

In view of Lemma 3.2, we can suppose that $H_{n}$ contains $\mathbb{Z}^{k+\ell} \times\{0\}^{m}$. (Indeed, $\left(A_{n} H_{n}\right)$ tends to $H$ and $\left(A_{n} H_{n}\right)^{\curlywedge}=\left(A_{n}^{t}\right)^{-1} H_{n}^{\curlywedge}$ tends to $W$.) We then claim that the orthogonal projection of $H_{n}$ onto $\{0\}^{k+\ell} \times \mathbb{R}^{m}$ is a discrete subgroup $\Gamma_{n}$ of systole (shortest vector) tending to infinity. Indeed, if $H_{n}$ contains an element of the form $\left(x_{n}, y_{n}, z_{n}\right)$ with $\left(z_{n}\right)$ bounded and nonzero for infinitely many $n$ 's, we can first multiply these elements by integers so that $\left\|z_{n}\right\| \geq 1$ for infinitely many $n$ 's, and then by translating by elements of $\mathbb{Z}^{k+\ell} \times\{0\}^{m}$, which is contained in $H_{n}$ by assumption, we can suppose $x_{n}$ and $y_{n}$ are bounded. Then at the limit, we obtain in $H$ an element whose third coordinate is nonzero, a contradiction. Thus $H_{n} \subset \mathbb{R}^{k} \times \mathbb{Z}^{\ell} \times \Gamma_{n}$, and the systole of $\Gamma_{n}$ tends to infinity. So

$$
H_{n}^{\curlywedge} \supset\{0\}^{k} \times \mathbb{Z}^{\ell} \times \Gamma_{n}^{\curlywedge} .
$$

By Proposition 3.4, $\Gamma_{n}^{\curlywedge}$ tends to $\mathbb{R}^{m}$. So $W \supset\{0\}^{k} \times \mathbb{Z}^{\ell} \times \mathbb{R}^{m}$ and we are done.

Proof of Theorem 1.1 It is explained by Protasov [25] how Theorem 3.1 implies Theorem 1.1; we do not repeat the full argument here, but the reader can complete the proof as follows:

- Show that the class $\mathcal{C}$ of LCA-groups $G$ for which the orthogonal map $\mathcal{S}(G) \rightarrow$ $\mathcal{S}\left(G^{\vee}\right)$ is continuous, is stable under taking 
- closed subgroups;

- Pontryagin dual;

- direct limits (namely if every open compactly generated subgroup of $G$ is in $\mathcal{C}$ then $G$ is in $\mathcal{C}$ ).

- Check that the smallest class of (isomorphism classes of) LCA-groups containing $\mathbb{R}^{d}$ for all $d$ and closed under the three operations above is the class of all LCAgroups.

\section{Dimension}

In this section, we have to switch from inductive to topological dimension and vice versa (see the reminder in Section 2.2), depending on the tools available. In case the two are known to coincide (see Proposition 2.2), we simply denote it by dim. Although our general results is in terms of topological dimension (because of the projective limit argument, see Proposition 2.2(1)), we have to detour through the inductive dimension. The following lemma is from Hurewicz and Wallman [17, Theorem VI.7].

Lemma 4.1 Let $f$ be a closed map between separable metrizable spaces. If all fibers of $f$ have dimension $\leq b$, then $\operatorname{indim}(X) \leq \operatorname{indim}(Y)+b$.

\section{Proposition 4.2}

$$
\operatorname{dim}\left(\mathcal{S}\left(\mathbb{R}^{d}\right)\right)=d^{2}
$$

Proof Since the set of lattices is an open subset of $\mathcal{S}\left(\mathbb{R}^{d}\right)$ and is a $d^{2}$-dimensional manifold, we have indim $\left(\mathcal{S}\left(\mathbb{R}^{d}\right)\right) \geq d^{2}$. Also, $\mathcal{S}\left(\mathbb{R}^{d}\right)$ has a natural finite partition into $\mathrm{GL}_{d}(\mathbb{R})$-orbits, each of which is a manifold of dimension $\leq d^{2}$. Therefore $\mathcal{S}\left(\mathbb{R}^{d}\right)$ is a separable metrizable space which is a union of countably many closed subspaces, each of which is homeomorphic to a closed ball in $\mathbb{R}^{k}$ for some $k \leq d^{2}$. By Engelking [12, Corollary 1.5.4], it follows that $\operatorname{indim}\left(\mathcal{S}\left(\mathbb{R}^{d}\right)\right) \leq d^{2}$.

Definition 4.3 Let $X$ be a locally compact space. Define $\operatorname{indim}_{\infty}(X)$ as the inductive dimension of the Alexandrov compactification of $X$ at the point $\infty$, that is,

$$
\operatorname{indim}_{\infty}(X)=\sup _{K}\left(1+\inf _{L}\{\operatorname{indim} \partial L: L \supset K\}\right),
$$

where $K, L$ range over compact subsets of $G$.

Lemma 4.4 Let $G$ be a metrizable LCA-group. Then $\operatorname{indim}_{\infty}(G)=0$ if $G$ is compact-by-discrete, and $\operatorname{indim}_{\infty}(G)=\operatorname{indim}(G)$ otherwise. 
Proof The statement remains the same if we replace $G$ by an open subgroup. Hence we can suppose $G=\mathbb{R}^{k} \times K$ with $K$ compact. Then the point at infinity has neighbourhoods with boundary of the form $S \times K$, where $S$ is a $(k-1)$-sphere. Observe that $S \times K$ has dimension $\leq(k-1)+\operatorname{indim}(K)$ if $k \geq 1$ and is empty if $k=0$. So if $k \geq 1$, we get $\operatorname{indim}_{\infty}(G) \leq k+\operatorname{indim}(K)=\operatorname{indim}(G)$, the latter equality using Lemma 2.9, and if $k=0$ we get $\operatorname{indim}_{\infty}(G)=0$.

Conversely, denoting by $D^{n}$ the closed $n$-disc, we use the fact that as a consequence of Lemma 2.9, $G$ contains a closed subset homeomorphic to $\mathbb{R}^{k} \times D^{n}$ for $n=\operatorname{indim}(K)$ (or for any $n$ if indim $(K)=\infty$ ). So if $k \geq 1$, the Alexandrov compactification of $G$ contains the Alexandrov compactification of $\mathbb{R}_{\geq 0} \times D^{n+k-1}$, which is a $(k+n)$-sphere passing through the point at infinity. $\operatorname{So~} \operatorname{indim}_{\infty}(G) \geq \operatorname{indim}(G)$.

Lemma 4.5 Let $G$ be a metrizable LCA-group. Then

$$
\operatorname{indim}(\mathcal{S}(G \times \mathbb{Z})) \leq \operatorname{indim}(\mathcal{S}(G))+\operatorname{dim}(G) .
$$

Proof Consider the natural map $p_{G \times\{0\}}: \mathcal{S}(G \times \mathbb{Z}) \rightarrow \mathcal{S}(G)$. The fiber of $H$ is homeomorphic to the set of partially defined homomorphisms $\mathbb{Z} \rightarrow G / H$, which is homeomorphic to the Alexandrov compactification of $G / H \times \mathbb{Z}_{>0}$. A point not at infinity in this space has dimension $\operatorname{indim}(G / H)$. The point at infinity has a basis of neighbourhood given by the complements of $K \times F$, for $K$ compact in $G / H$ and $F$ finite in $\mathbb{Z}_{>0}$, so if we restrict to $K$ with indim $\partial K \leq \operatorname{indim}_{\infty}(G / H)-1$, we deduce that the dimension of the fiber is $\leq \max \left(\operatorname{indim}(G / H), \operatorname{indim}_{\infty}(G / H)\right)$. By Lemma 4.1, we get

$\operatorname{indim}(\mathcal{S}(G \times \mathbb{Z})) \leq \operatorname{indim}(\mathcal{S}(G))+\sup _{H \in \mathcal{S}(G)}\left(\max \left(\operatorname{indim}(G / H), \operatorname{indim}_{\infty}(G / H)\right)\right)$.

Now by Lemma 4.4, $\operatorname{indim}_{\infty}(G / H) \leq \operatorname{indim}(G / H)$, and by Lemma 2.9,

$$
\operatorname{indim}(G / H) \leq \operatorname{indim}(G) .
$$

Theorem 4.6 The inductive, or topological, dimension of $\mathcal{S}\left(\mathbb{R}^{k} \times \mathbb{Z}^{\ell} \times(\mathbb{R} / \mathbb{Z})^{m} \times F\right)$, for $F$ finite, is $(k+\ell)(k+m)$, and is achieved by a piece of manifold.

Proof Since $\mathcal{S}\left(\mathbb{R}^{k} \times \mathbb{Z}^{\ell} \times(\mathbb{R} / \mathbb{Z})^{m} \times F\right)$ is compact and metrizable, the two notions of dimension coincide (Proposition 2.2(1)) so we can work with the inductive dimension.

First, we have to embed a $(k+\ell)(k+m)$-dimensional manifold into $\mathcal{S}\left(\mathbb{R}^{k} \times\right.$ $\left.\mathbb{Z}^{\ell} \times(\mathbb{R} / \mathbb{Z})^{m}\right)$. It will be convenient to rewrite the group as $\mathbb{Z}^{\ell} \times \mathbb{R}^{k} \times(\mathbb{R} / \mathbb{Z})^{m}$. We consider the action of the group of automorphisms of the form $(x, y, z) \mapsto$ $(x, A y+B x, z+C x+D y)$, where $A \in \operatorname{GL}\left(\mathbb{R}^{k}\right), B \in \operatorname{Hom}\left(\mathbb{Z}^{\ell}, \mathbb{R}^{k}\right) \simeq \mathbb{R}^{\ell k}$, 
$C \in \operatorname{Hom}\left(\mathbb{Z}^{\ell},(\mathbb{R} / \mathbb{Z})^{m}\right) \simeq(\mathbb{R} / \mathbb{Z})^{\ell m}, D \in \operatorname{Hom}\left(\mathbb{R}^{k},(\mathbb{R} / \mathbb{Z})^{m}\right) \simeq \mathbb{R}^{k m}$. Consider the subgroup $\mathbb{Z}^{k+\ell}$ : its stabilizer is discrete, so its orbit is $(k+\ell)(k+m)$-dimensional; this is the desired piece of manifold and provides the easy inequality.

Conversely, to obtain that the inductive dimension is bounded as given, we first reduce the case from $G \times F$ to $G$. Each fiber of the map $\mathcal{S}(G \times F) \rightarrow \mathcal{S}(G)$ can be identified to the set of partial homomorphisms from $F$ to some quotient of $G$, hence is finite. So by Lemma 4.1, we have $\operatorname{indim}(\mathcal{S}(G \times F)) \leq \operatorname{indim}(\mathcal{S}(G))$, the other inequality being trivial.

By Lemma 4.5, we obtain by induction that

$$
\operatorname{indim}\left(\mathcal{S}\left(\mathbb{R}^{k} \times \mathbb{Z}^{\ell}\right)\right) \leq \operatorname{indim}\left(\mathcal{S}\left(\mathbb{R}^{k}\right)\right)+k l .
$$

By Pontryagin-Chabauty duality,

$$
\operatorname{indim}\left(\mathcal{S}\left(\mathbb{R}^{k} \times(\mathbb{R} / \mathbb{Z})^{m}\right)\right) \leq \operatorname{indim}\left(\mathcal{S}\left(\mathbb{R}^{k}\right)\right)+k m,
$$

and by a second induction,

$$
\operatorname{indim}\left(\mathcal{S}\left(\mathbb{R}^{k} \times \mathbb{Z}^{\ell} \times(\mathbb{R} / \mathbb{Z})^{m}\right)\right) \leq \operatorname{indim}\left(\mathcal{S}\left(\mathbb{R}^{k}\right)\right)+k m+\ell(k+m),
$$

and finally by Proposition 4.2

$$
\operatorname{indim}\left(\mathcal{S}\left(\mathbb{R}^{k} \times \mathbb{Z}^{\ell} \times(\mathbb{R} / \mathbb{Z})^{m}\right)\right) \leq(k+\ell)(k+m) .
$$

We can now state the general result (see Lemma 2.9 and Corollary 2.10 for interpretations of $\operatorname{tdim}(G)$ and $\left.\operatorname{tdim}\left(G^{\vee}\right)\right)$.

Theorem 4.7 Let $G$ be an LCA-group and $\delta=\operatorname{tdim}(G) \operatorname{tdim}\left(G^{\vee}\right) \in\{0,1, \ldots, \infty\}$ (where $0 \infty=\infty 0=0$ ). The topological dimension of $\mathcal{S}(G)$ is given by

$$
\operatorname{tdim}(\mathcal{S}(G))=\delta
$$

It is achieved by a piece of manifold, that is, if $\delta<\infty$ then $\mathcal{S}(G)$ contains a closed subset homeomorphic to the closed $\delta$-ball, with $\delta=\operatorname{tdim}(G) \operatorname{tdim}\left(G^{\vee}\right)$; in case $\delta=\infty$, it contains closed balls of arbitrary large dimension.

Proof For the inequality $\geq$, Lemma 2.11 reduces to $G=\mathbb{Z}^{(I)} \times(\mathbb{R} / \mathbb{Z})^{J} \times \mathbb{R}^{k}$. If $I, J$ are finite, then by Theorem 4.6 we get a piece of $n$-manifold, where $n=$ $(k+\# I)(k+\# J)$. If $(k+\# I)(k+\# J) \neq 0$ and either $I$ or $J$ is infinite, the same argument allows to find pieces of manifolds of arbitrary large dimension.

Let us prove $\leq$. If either $\operatorname{tdim}(G)$ or $\operatorname{tdim}\left(G^{\vee}\right)$ is zero, then $G$ is totally disconnected, or elliptic, and then we know by Corollary 7.5 that $\mathcal{S}(G)$ is totally disconnected, so is zero-dimensional. 
We henceforth assume $\operatorname{tdim}(G) \operatorname{tdim}\left(G^{\vee}\right)$ nonzero. If either $\operatorname{tdim}(G)$ or $\operatorname{tdim}\left(G^{\vee}\right)$ is infinite there is nothing to prove, so we suppose both finite and nonzero.

In case $G$ is a compactly generated Lie group, in view of Proposition 2.3, the result is given by Theorem 4.6.

First assume that $G$ is a Lie group. Then in view of Corollary 2.10, $G$ has an open, compactly generated subgroup $M$ such that for every subgroup containing $N$, we have $\operatorname{tdim}\left(N^{\vee}\right)=\operatorname{tdim}\left(G^{\vee}\right)$. Now $\mathcal{S}(G)$ is the projective limit of $\mathcal{S}(N)$, when $N$ ranges over subgroups containing $M$, which is of $\operatorname{dimension} \operatorname{tdim}(G) \operatorname{tdim}\left(G^{\vee}\right)$. By Proposition 2.2(4), it follows that $\operatorname{tdim}(\mathcal{S}(G)) \leq \operatorname{tdim}(G) \operatorname{tdim}\left(G^{\vee}\right)$.

Now by Pontryagin-Chabauty duality, the result holds when $G$ is a compactly generated LCA-group. Repeating a second time the projective limit argument, we obtain the result for a general LCA-group.

\section{Isolated points}

In this section, we prove Theorem 1.7.

Lemma 5.1 The subgroup $\{0\}$ is isolated in $\mathcal{S}(G)$ if and only if $G \simeq T \times D$, with $T \simeq(\mathbb{R} / \mathbb{Z})^{k}$ a torus, and $D$ discrete Artinian.

Proof Suppose $\{0\}$ isolated. Then $G$ does not contain any discrete copy of $\mathbb{Z}$, so is elliptic. Let $U$ be a compact open subgroup. Then $\{0\}$ is isolated in $\mathcal{S}(U)$, so $U^{\vee}$ is isolated in $\mathcal{S}\left(U^{\vee}\right)$. As $U^{\vee}$ is discrete, this means that $U^{\vee}$ is finitely generated (see the paper [9] by the author, Guyot and Pitsch), so replacing $U$ by a finite index subgroup, we can suppose that $U$ is a torus. As $U$ is open and divisible, it has a direct factor $D$ in $G$, which is discrete. As $\{0\}$ is isolated in $\mathcal{S}(D)$ as well, we deduce from [9, Lemma 4.1] that $D$ is Artinian.

Conversely, let us assume that $T$ is a torus, $D$ is Artinian, $G=T \times D$, and let us prove that $\{0\}$ is isolated in $\mathcal{S}(G)$. If $D$ is finite, then the Pontryagin dual is a finitely generated discrete abelian group, so $G^{\vee}$ is isolated in $\mathcal{S}\left(G^{\vee}\right)$, so $\{0\}$ is isolated in $\mathcal{S}(G)$. In general, if $D_{\text {prime }}$ is the subgroup of $D$ generated by elements of prime order, it is easy to check that $T \times D_{\text {prime }}-\{0\}$ is a discriminating subset of $T \times D$, that is, every non-trivial subgroup of $T \times D$ has non-trivial intersection with $T \times D_{\text {prime }}$. Therefore since $D_{\text {prime }}$ is finite, this reduces to the case when $D$ is finite and we are done.

Lemma 5.2 The subgroup $G$ is isolated in $\mathcal{S}(G)$ if and only if $G \simeq A \times P$, with $A$ a finitely generated abelian group, and $P$ an adic group. 
Proof Follows from Lemma 5.1 by Pontryagin-Chabauty duality.

In the discrete setting, a necessary and sufficient condition for a subgroup $H$ to be isolated in $\mathcal{S}(G)$ is that $H$ be isolated in $\mathcal{S}(H)$ and $\{0\}$ be isolated in $\mathcal{S}(G / H)$. In the locally compact setting, this does not hold any longer, although there is essentially a unique obstruction, given by the following lemma.

Lemma 5.3 The subgroup $\mathbb{Z} \times\{0\}$ is not isolated in $\mathcal{S}(\mathbb{Z} \times \mathbb{R} / \mathbb{Z})$.

Proof It is part of the continuous family of subgroups $\langle(1, t)\rangle$ for $t \in \mathbb{R} / \mathbb{Z}$.

Proof of Theorem 1.7 Suppose that $H$ is isolated. So $H$ is isolated in $\mathcal{S}(H)$ and $\{0\}$ is isolated in $\mathcal{S}(G / H)$. By Lemmas 5.1 and 5.2, we deduce that $H \simeq A \times P$, $G / H \simeq T \times D$, with $A$ finitely generated abelian, $P$ adic, $T$ torus, $D$ Artinian. Assume that simultaneously $A$ is infinite and $T$ is non-trivial. Then we can embed $\mathcal{S}(\mathbb{Z} \times \mathbb{R} / \mathbb{Z})$ into $\mathcal{S}(G)$, mapping $\mathbb{Z}$ to $H$. But $\mathbb{Z}$ is not isolated in $\mathcal{S}(\mathbb{Z} \times \mathbb{R} / \mathbb{Z})$ by Lemma 5.3, so $H$ is not isolated.

Conversely, assume that $H \simeq A \times P, G / H \simeq D, A$ finitely generated abelian, $P$ adic, $D$ Artinian. Suppose $L$ is close enough to $H$. As $H$ is clopen, this means that $L \cap H$ is close to $H$, and as $H$ is isolated in $\mathcal{S}(H)$ by Lemma 5.2, this implies that $L$ contains $H$. Now using that $\{0\}$ is isolated in $\mathcal{S}(G / H)$, we deduce that $L=H$, so $H$ is isolated. The second case is equivalent by Pontryagin-Chabauty duality.

\section{Some natural maps}

Let $G$ be an LCA-group. If $\Omega$ is an open subgroup, then the map $\mathcal{S}(G) \rightarrow \mathcal{S}(\Omega)$ mapping $H$ to $H \cap \Omega$ is obviously continuous and surjective, and we refer to it as the natural map $i_{\Omega}: \mathcal{S}(G) \rightarrow \mathcal{S}(\Omega)$.

By duality, if $K$ is a compact subgroup, then the projection map $p_{K}: \mathcal{S}(G) \mapsto \mathcal{S}(G / K)$ mapping $H$ to $(H+K) / K$, is continuous and surjective. (When $\Omega$ is not open or $K$ non-compact, these maps often fail to be continuous, see Protasov and Tsybenko [27] for a discussion.)

Note that if $K \subset \Omega$, the maps $i_{\Omega}$ and $p_{K}$ commute in an obvious way, and the composition map $\rho_{\Omega, K}: \mathcal{S}(G) \rightarrow \mathcal{S}(\Omega / K)$ maps $H$ to $((H+K) \cap \Omega) / K=((H \cap$ $\Omega)+K) / K$.

If $K^{\prime} \subset K \subset \Omega \subset \Omega^{\prime}$, then obviously

$$
\rho_{\Omega, K}^{G}=\rho_{\Omega / K^{\prime}, K / K^{\prime}}^{\Omega^{\prime} / K^{\prime}} \circ \rho_{\Omega^{\prime}, K^{\prime}}^{G}
$$


where we write $\rho_{\Omega, K}^{G}$ for $\rho_{\Omega, K}$ in order to mention $G$. Therefore, if we have a net $\left(K_{i}, \Omega_{i}\right)$, filtering in the sense that if $j \geq i$ then $K_{j} \subset K_{i} \subset \Omega_{i} \subset \Omega_{j}$, we obtain a natural map

$$
\mathcal{S}(G) \rightarrow \underset{\lim }{\leftarrow}\left(\Omega_{i} / K_{i}\right)
$$

This map has dense image, hence is surjective. It is injective provided $\bigcap K_{i}=\{0\}$ and $\bigcup \Omega_{i}=G$, in which case it is a homeomorphism.

We can play another game with these maps. Consider the diagonal map

$$
i_{\Omega} \times p_{K}: \mathcal{S}(G) \rightarrow \mathcal{S}(\Omega) \times \mathcal{S}(G / K) .
$$

This map need not be surjective. It is interesting because its fibers have a very special form.

Proposition 6.1 Let $G$ be an LCA-group, $K$ a compact subgroup, $\Omega$ an open subgroup containing $K$. Consider the map

$$
\begin{aligned}
\mathcal{S}(G) & \rightarrow \mathcal{S}(\Omega) \times \mathcal{S}(G / K) \\
H & \mapsto(H \cap \Omega,(H+K) / K)
\end{aligned}
$$

as above. Then the fiber of $(R, M / K)$ is either empty, or homeomorphic to the compact group

$$
\operatorname{Hom}((M+\Omega) / \Omega, K /(K \cap R))
$$

(in particular it is homogeneous).

Proof If $H \cap \Omega=R$ is assumed, then obviously $H+K=M$ is equivalent to $H+K+R=M$. So we can suppose that $R=0$ without changing the statement. Also, we can suppose that $M=G$. So we have to prove that the set $\mathcal{F}$ of subgroups $H$ with $H \cap \Omega=\{0\}$ and $H+K=G$ is either empty or homeomorphic to $\operatorname{Hom}(G / \Omega, K)$. Suppose there is at least one such group $V$. Then since $K \subset \Omega$ we get $G=V \oplus K=$ $V \oplus \Omega$, so $K=\Omega$ (in the original group this means that $\Omega \cap M=K+R$ when the fiber is non-empty) and $V$ is isomorphic to $G / \Omega$. We see that $H \in \mathcal{F}$ if and only if it is the graph of a homomorphism $V \rightarrow K$. So $\mathcal{F}$ is homeomorphic to $\operatorname{Hom}(G / \Omega, K)$.

\section{Groups with $R=0$ : study of connected components}

In this section and the next one, we study connectedness of $\mathcal{S}(G)$. The study of path-connectedness and local connectedness of $G$ itself was done by Dixmier [11] and seems to be, to a large extent, fairly unrelated. 
Lemma 7.1 Let $G$ be an LCA-group. If $G$ is either discrete or compact, then $\mathcal{S}(G)$ is totally disconnected.

Proof If $G$ is discrete, then $\mathcal{S}(G)$ is a closed subset of $2^{G}$ and is therefore totally disconnected. By duality, we deduce the same result if $G$ is compact.

Proposition 7.2 If $R(G)=0$ and $G$ is infinite, then $\mathcal{S}(G)$ has infinitely many connected components.

Proof If $G$ is discrete, then $\mathcal{S}(G)$ is totally disconnected and infinite and we are done.

Assume $G$ non-discrete. By Lemma 2.4, there exists a compact open subgroup $M$ in $G$. The natural map (see Section 6) $i_{M}: \mathcal{S}(G) \rightarrow \mathcal{S}(M)$ is continuous and surjective. Since $M$ is compact and infinite, its Pontryagin dual is discrete and infinite (see Section 2.5), so $\mathcal{S}\left(M^{\vee}\right)$ is infinite and totally disconnected, and is homeomorphic to $\mathcal{S}(M)$ by Pontryagin-Chabauty duality (Theorem 1.1).

Lemma 7.3 Suppose that $G$ is either elliptic, or totally disconnected. Then $\mathcal{S}(G)$ is totally disconnected.

Proof Let $K$ be a compact open subgroup. Proposition 6.1 provides a continuous map from $\mathcal{S}(G)$ to $\mathcal{S}(K) \times \mathcal{S}(G / K)$, which is totally disconnected by Lemma 7.1. Therefore any connected subset of $\mathcal{S}(G)$ is contained in a fiber of this map, and by Proposition 6.1 again, any such nonempty fiber is homeomorphic to $\operatorname{Hom}(D, Q)$ for some subgroup $D$ of $G / K$ and some quotient $Q$ of $K$. If $G$ is totally disconnected, so is $Q$, and therefore $\operatorname{Hom}(D, Q)$ is totally disconnected, so $\mathcal{S}(G)$ is totally disconnected. By duality, the same conclusion holds if $G$ is elliptic.

Recall that $E_{G}$ denotes the elliptic subgroup of an LCA-group $G$.

Theorem 7.4 Let $G$ be an LCA-group with $R(G)=0$. For any $H \in \mathcal{S}(G)$, set $N=H+G_{0}$ and $L=H \cap E_{G}$. Then the connected component of $H$ in $\mathcal{S}(G)$ consists of the subgroups $H^{\prime}$ such that $H^{\prime}+G_{0}=N$ and $H^{\prime} \cap E_{G}=L$, and is homeomorphic to the compact group

$$
\operatorname{Hom}\left(\left(N+E_{G}\right) / E_{G}, G_{0} /\left(G_{0} \cap L\right)\right) \text {. }
$$


Proof Set $K=G_{0}, \Omega=E_{G}$. Since $R(G)=0, K$ is compact and $\Omega$ is open. Consider the continuous map $i_{\Omega} \times p_{K}: \mathcal{S}(G) \rightarrow \mathcal{S}\left(E_{G}\right) \times \mathcal{S}\left(G / G_{0}\right)$. By Proposition 6.1, the nonempty fibers are homeomorphic to the compact group

$$
\operatorname{Hom}\left(\left(N+E_{G}\right) / E_{G}, G_{0} /\left(G_{0} \cap L\right)\right) .
$$

By Lemma 7.3, the target space $\mathcal{S}\left(E_{G}\right) \times \mathcal{S}\left(G / G_{0}\right)$ is totally disconnected, and by Lemma 2.6 the fibers are connected. The theorem follows.

Corollary 7.5 Let $G$ be an LCA-group. Equivalences:

- $\mathcal{S}(G)$ is totally disconnected;

- $G$ is either elliptic or totally disconnected.

Proof The reverse implication is Lemma 7.3. Conversely assume $\mathcal{S}(G)$ is totally disconnected. It is trivial (and a particular case of several already proved results) that this implies $R(G)=0$. If $G$ is not elliptic, it contains a closed subgroup $H$ isomorphic to $\mathbb{Z}$. Applying Theorem 7.4, we obtain that the connected component of $H$ in $\mathcal{S}(G)$ is homeomorphic to $G_{0}$, therefore is not reduced to a point unless $G$ is totally disconnected.

Definition 7.6 A point $x$ of a topological space $X$ is

- path-rigid if its path-connected component is reduced to $\{x\}$;

- rigid if its connected component is reduced to $\{x\}$.

Plainly, rigid implies path-rigid. If $\{x\}$ is an intersection of clopen subsets, then $x$ is rigid; the converse holds when $X$ is compact, by Bourbaki [4, II.4.4, Proposition 6].

Let us denote by $D_{\mathrm{tf}}$ the quotient of the discrete group $D$ by its torsion subgroup.

Corollary 7.7 Let $H$ be a closed subgroup of the LCA-group $G$. Equivalences:

(i) $H$ is rigid in $\mathcal{S}(G)$;

(ii) $H$ is path-rigid in $\mathcal{S}(G)$;

(iii) $R(G)=0$, and either $H$ is elliptic or $G / H$ is totally disconnected.

Proof $\quad$ (i) $\Rightarrow$ (ii) is trivial.

(ii) $\Rightarrow$ (i) By Proposition 8.2, $R(G)=0$, so we can apply the theorem, which says that the connected component of $H$ is homeomorphic to a compact group. So we can apply Lemma 2.5. 
(i) $\Leftrightarrow$ (iii). The condition $R(G)=0$ follows from (iii) by definition, and from (i) by Proposition 8.2. So assuming $R(G)=0$, the equivalence is obtained as follows. Set $N=H+G_{0}$ and $L=H \cap E_{G}$. By the theorem, the path component of $H$ is homeomorphic to $\operatorname{Hom}\left(\left(N+E_{G}\right) / E_{G}, G_{0} /\left(G_{0} \cap L\right)\right)$. Since $\left(N+E_{G}\right) / E_{G}$ is a torsion-free discrete group and $G_{0} /\left(G_{0} \cap L\right)$ is a connected compact group, by Lemma 2.6, the connected group $\operatorname{Hom}\left(\left(N+E_{G}\right) / E_{G}, G_{0} /\left(G_{0} \cap L\right)\right)$ is trivial if and only if either $\left(N+E_{G}\right) / E_{G}$ or $G_{0} /\left(G_{0} \cap L\right)$ is trivial, or equivalently, either $N \subset E_{G}$ or $G_{0} \subset L$, that is, either $H+G_{0} \subset E_{G}$ or $G_{0} \subset E_{G} \cap H$. Since $R(G)=0$, we have $G_{0} \subset E_{G}$, so the previous condition is equivalent to either $H \subset E_{G}$ or $G_{0} \subset H$, which means that either $H$ is elliptic or $G / H$ is totally disconnected.

We now give a structure result for LCA-groups for which $\pi_{0}(\mathcal{S}(G))$ is countable, or equivalently scattered. We first recall the following classical proposition, which has reappeared several times in the literature.

Proposition 7.8 Let $G$ be an LCA-group.

(1) If $G$ is discrete, then $\mathcal{S}(G)$ is countable if and only if $G$ lies in an extension

$$
1 \rightarrow Z \rightarrow G \rightarrow A \rightarrow 1
$$

where $Z$ is a finitely generated abelian group, and $A \simeq \mathbb{C}_{m} \infty$ for some $m \geq 1$.

(2) If $G$ is compact, then $\mathcal{S}(G)$ is countable if and only if $G$ lies in an extension

$$
1 \rightarrow P \rightarrow G \rightarrow T \rightarrow 1
$$

where $T$ is a compact Lie group and $P \simeq \mathbb{Z}_{m}$ for some $m \geq 1$.

Note that in the statement above, $m$ is not assumed prime. As far as we know (1) was first proved by Boyer [5] and (2) immediately follows by Pontryagin duality.

Theorem 7.9 Let $G$ be a compact-by-discrete LCA-group. Equivalences:

(a) $\pi_{0}(\mathcal{S}(G))$ is scattered.

(b) $\pi_{0}(\mathcal{S}(G))$ is countable.

(c) $G$ has a compact open subgroup $M$ such that $\mathcal{S}(M)$ and $\mathcal{S}(G / M)$ are countable, and one of the following condition holds

(1) $M$ is finite;

(2) $G / M$ is finite;

(3) $M$ is profinite and $G / M$ is torsion;

(4) $M$ is virtually connected and $G / M$ has finite torsion. 
(d) One of the following condition holds

(1') $G$ is discrete with countably many subgroups (see Proposition 7.8);

(2') $G$ is compact with countably many subgroups (dual to the previous case);

(3') $G=\mathbb{Q}_{\ell} \times \mathbb{Z}_{m} \times \mathbb{C}_{n} \infty \times F$, where $F$ is finite, $\ell, m, n \geq 1, \ell$ is prime with $m n$.

(4') $G=K \times D \times F$ with $D$ torsion-free discrete, $K$ compact connected, $F$ finite, and $\mathcal{S}(D)$ and $\mathcal{S}(K)$ are countable.

Note that in Cases (1),(2),(3), $\mathcal{S}(G)$ is countable itself. In (4), $\mathcal{S}(G)$ is not totally disconnected unless $G$ is compact or discrete.

We need some preliminary lemmas.

Lemma 7.10 Let $G$ be an LCA-group with a compact open subgroup $M$. Suppose that $G$ is divisible and torsion-free. Assume that $M$ is adic and $G / M$ is discrete artinian. Then $G$ is local.

Proof Let $S$ be the (finite) set of primes occurring in the canonical decomposition of $M$ (as a product of pro- $p$-groups for distinct primes $p$ ). Since this decomposition is canonical, it does not depend on $M$ and it follows that $G$ also inherits such a decomposition. Hence we can reduce to the case when $S=\{p\}$. So $M$ is isomorphic to $\mathbb{Z}_{p}^{m}$ for some $m$. So we have a continuous embedding $q: M \rightarrow \mathbb{Q}_{p}^{m}$. Denote by $i$ the inclusion of $M$ into $G$. Since $G$ is divisible as an abstract abelian group, there exists an abstract homomorphism $f: \mathbb{Q}_{p}^{m}$ such that $f \circ q=i$. Since the restriction of $f$ to the open subgroup $q(M)=\mathbb{Z}_{p}^{m}$ is continuous, $f$ is continuous as well. The kernel of $f$ has trivial intersection with $\mathbb{Z}_{p}^{m}$, hence is trivial, that is, $f$ is injective. Besides, the image $f\left(\mathbb{Q}_{p}^{m}\right)$ contains $M$, hence is open, and is divisible. So it has a direct factor $\Gamma$ in $G$, necessarily discrete. Now $\Gamma$ is torsion-free as a subgroup of $G$, and artinian as a subgroup of $G / M$. So $\Gamma=\{0\}$.

Lemma 7.11 Let $G$ be an LCA-group with a compact open subgroup $M$. Assume that $M$ is adic and $G / M$ is discrete artinian. Then we can write $G$ as $Q \times Z \times D \times F$ with $Q$ local, $Z$ torsion-free adic, $D$ discrete artinian, and $F$ finite.

Proof We can suppose that $M$ is torsion-free. Let $T$ be the torsion subgroup in $G$. Since $T \cap M=\{0\}$, we know that $T$ is a discrete subgroup. Moreover $T$ embeds into $G / M$, so $T$ is Artinian. Let $S$ be the divisible part of $T$, which has finite index in $T$. Since $T_{d} \cap M=\{0\}, D$ has a direct factor $G_{1}$ in $G$ containing $M$. Working similarly in the Pontryagin dual of $G_{1}$, we write $G=Z \times D \times G_{2}$ where $Z$ is torsion-free adic, and both $G_{2}$ and its Pontryagin dual have finite torsion. Since the Pontryagin dual 
of $G_{2}$ has finite torsion, the index $\left[G_{2}: n G_{2}\right]$ is bounded independently of $n$. So the divisible part $\bigcap_{n} n ! G_{2}$ of $G_{2}$ has finite index and we can write $G_{2}=F \times G_{3}$ with $F$ finite, $G_{3}$ divisible and torsion-free (and satisfying the hypotheses of the lemma). We have $G=Z \times D \times F \times G_{3}$. By Lemma 7.10, $G_{3}$ is local.

\section{Proof of Theorem 7.9}

- (b) $\Rightarrow$ (a) follows from the general fact that a countable compact Hausdorff space is scattered (see Section 2.3).

- (c) $\Rightarrow$ (b). First note that the result is clear if (1) or (2) holds, since then $\mathcal{S}(G)$ is countable.

Consider the natural map (introduced in Section 6)

$$
i_{M} \times p_{M}: \mathcal{S}(G) \rightarrow \mathcal{S}(M) \times \mathcal{S}(G / M) .
$$

By Proposition 6.1, each non-empty fiber is of the form $\operatorname{Hom}(N, Q)$ with $N \leq G / M$ and $Q \leq M$. If (3) holds, then $Q$ has finite torsion $Q_{t}$ and the divisible part $N_{d}$ of $N$ has finite index, so $\operatorname{Hom}(N, Q)=\operatorname{Hom}\left(N / N_{d}, Q_{t}\right)$ is finite. So the fibers are finite and $\mathcal{S}(G)$ is countable.

- (a) $\Rightarrow$ (c). Suppose that $\pi_{0}(\mathcal{S}(G))$ is scattered and let $M$ be a compact open subgroup of $G$. Then $\pi_{0}(\mathcal{S}(M))$ and $\pi_{0}(\mathcal{S}(G / M))$ are scattered as well, hence countable (Lemma 7.1). Moreover, for every fiber $\mathcal{F}$ of the natural map (7-1), we have $\pi_{0}(\mathcal{F})$ scattered. By Proposition 6.1, these fibers are homeomorphic to compact groups. Thus $\pi_{0}(\mathcal{F})$ is both scattered and homeomorphic to a compact Lie group, so is finite, that is, the fibers have finitely many components. Therefore for all closed subgroups $0 \leq L \leq M \leq N \leq G$, we have $\operatorname{Hom}(N / M, M / L)$ virtually connected.

- Suppose $\mathbb{Z}$ embeds into $G / M$. Then taking $N$ such that $N / M \simeq \mathbb{Z}$, we have $\operatorname{Hom}(N / M, M)=M$ virtually connected.

- In a dual way, if $M$ is not profinite, that is, if $\mathbb{Z}$ does not embed into $M^{\vee}$, then $(G / M)^{\vee}$ is virtually connected, that is, $G / M$ has finite torsion.

Therefore one of the following holds

- $M$ is finite (Case (1))

- $M$ is infinite.

* $G / M$ is not torsion. Then $M$ is virtually connected; as it is infinite, it is not profinite, and hence $G / M$ has finite torsion. This is Case (4).

* $G / M$ is torsion. We again discuss.

- $M^{\vee}$ is not torsion. Then $(G / M)^{\vee}$ is virtually connected, so $G / M$ has finite torsion, so is finite (Case (2)). 


\section{- $M^{\vee}$ is torsion, so $M$ is profinite (Case (3)).}

- (c) $\Rightarrow($ d). Under the assumption that $\mathcal{S}(M)$ and $\mathcal{S}(G / M)$ are countable, let us prove (i) $\Rightarrow$ (i') for $i=1 \ldots 4$. For $i=1,2$ there is nothing to prove.

Suppose (4). We can suppose that $M$ is connected. Hence it is divisible, so has a direct factor $H$ in $G$. Then $H$ is discrete and finite-by-(torsion-free). So $H^{\vee}$ is connected-by-finite, and again the connected component, by the same argument, has a direct factor. This means that $H$ is the direct product of a finite group by a torsion-free group.

Suppose (3). Since $G / M$ is discrete torsion and $\mathcal{S}(G / M)$ is countable, $G / M$ is artinian of the form $\mathbb{C}_{n} \infty \times F_{1}$ with $F_{1}$ finite and $n$ square-free (see Boyer [5]). Dually, $G / M$ is adic of the form $\mathbb{Z}_{m} \times F_{2}$ with $F_{2}$ finite and $m$ square-free. By Lemma 7.11, we can write $G=Q \times Z \times D \times F$ with $Q$ local, $Z$ torsion-free adic, $D$ discrete artinian, and $F$ finite. Because of the special form of $M$ and $G / M$, We can write $Q, Z$ and $D$ in the desired form.

\section{Connectedness when $R \geq 1$}

In [26], Protasov and Tsybenko show that if $G=\{1\}$ is a locally compact group such that $\mathcal{S}(G)$ is connected, then $G$ contains a closed copy of $\mathbb{R}$. They show the converse holds for abelian groups; however their proof relies on the statement that for every locally compact group $G$, we have $\mathcal{S}(\mathbb{R} \times G)$ is connected. This is not true (see Proposition 8.6); however their proof can probably be fixed when $G$ is abelian; nevertheless their proof does not yield path-connectedness in the case of compactly generated Lie groups, as they typically show that two points are in the same connected component by showing the second one belongs to the closure of a path emanating from the first one.

Consider a locally compact group $G=\mathbb{R}^{k} \times H$ and $L$ a closed subgroup of $G$. Denote by $M$ the closure of the projection of $L$ on $H$. We consider the automorphism $\tau_{\lambda}(x, h)=(\lambda x, h)$. Consider the path $\tau_{\lambda-1}(L)$ for $\lambda \in[1,+\infty[$.

Lemma 8.1 Consider a group $G=\mathbb{R}^{k} \times H$ as above, with $k \geq 1$, where the locally compact group $H$ has a compact open subgroup (but need not be abelian). Let $L$ be a closed subgroup of $G$. Denote by $L_{1}$ the closure of the projection of $L$ on $H$. Then for some vector subspace $W$ of $\mathbb{R}^{k}, \tau_{\lambda-1}(L)$ tends to $W \times L_{1}$ when $\lambda$ tends to $+\infty$.

Proof Let $M$ denote any accumulation point of $\left(\tau_{\lambda-1}(L)\right)$. It is straightforward that $\{0\} \times L_{1} \subset M \subset \mathbb{R}^{k} \times L_{1}$. 
Let us begin by the case when $H$ is compact. Let $W$ be the vector space generated by the projection of $L$ on $\mathbb{R}^{k}$. Obviously $M \subset W \times L_{1}$. Take $v \in W$ and $\lambda_{i} \rightarrow+\infty$. Then $\lambda_{i} v$ is at bounded distance, say $\leq r$, of some element in the projection of $L$. That is, we can find $\left(w_{i}, h_{i}\right)$ in $L$ with $\left\|w_{i}-\lambda_{i} v\right\| \leq r$. So $\left\|\lambda_{i}^{-1} w_{i}-v\right\| \rightarrow 0$. So $\tau_{\lambda_{i}^{-1}}\left(w_{i}, h_{i}\right)$ accumulates to $(v, h)$ for some $h \in L_{1}$, by compactness of $H$. Therefore, since we know that $\{0\} \times L_{1}$ is contained in $M$, we deduce that $(v, 0)$ is contained in $M$. Since $v$ is arbitrary, $M=W \times L_{1}$.

In general, $H$ has a compact open subgroup $K$, and by the above, if $W$ is the vector space generated by the projection of $L \cap\left(\mathbb{R}^{k} \times K\right)$ on $\mathbb{R}^{k}$, then we obtain that $\tau_{\lambda-1}(L) \cap\left(\mathbb{R}^{k} \times K\right) \rightarrow W \times\left(L_{1} \cap K\right)$. As $M$ has to be of the form $F \times L_{1}$ for some closed subgroup $F$, we deduce that $F=W$ and $\tau_{\lambda-1}(L)$ tends to $W \times L_{1}$.

Proposition 8.2 Let $G=\mathbb{R}^{k} \times H$ be a locally compact group, where $H$ has a compact open subgroup. Let $L$ be a closed subgroup of $G$ and let $L_{1}$ be the closure of the projection of $L$ on $H$. Then the path-connected component of $L$ in $\mathcal{S}(G)$ contains $\mathcal{S}\left(\mathbb{R}^{k}\right) \times\left\{L_{1}\right\}$. In particular, if $k \geq 1$, then $L$ is not path-rigid (see Definition 7.6).

Proof This follows from Lemma 8.1, and path-connectedness of $\mathcal{S}\left(\mathbb{R}^{k}\right)$ (which is an easy exercise).

Define an LCA-group as circular if it is discrete and has an injective homomorphism into $\mathbb{R} / \mathbb{Z}$ (for instance, $\mathbb{R} / \mathbb{Z}$ with the discrete topology is circular). Define an LCA-group $G$ to be polycircular if it has a composition series

$$
\{0\}=G_{0} \subset G_{1} \subset \cdots \subset G_{n}=G
$$

such that each $G_{i} / G_{i-1}$ is circular, and metacircular if it can be written as $\mathbb{R}^{k} \times$ $H$, where $H$ has a compact open subgroup $M$ such that both $H / M$ and $M^{\vee}$ are polycircular. For instance, every compactly generated Lie group is metacircular. Also, $\mathbb{Q}_{p}$ is metacircular, but the infinite direct product $(\mathbb{Z} / 2 \mathbb{Z})^{\mathbb{Z}}$ is not.

Lemma 8.3 Let $H$ denote an LCA-group such that either $H$ or $H^{\vee}$ is circular. In $\mathcal{S}\left(\mathbb{R}^{k} \times H\right)$, if $k \geq 1$, we can join $\{0\} \times H$ to $\{0\}$ by a path.

Proof It is enough to prove the lemma for $k=1$, since then we can join $\mathbb{R}^{\ell} \times H$ to $\mathbb{R}^{\ell-1} \times H$ by a path and concatenate all those paths.

- $\quad H$ has a continuous injection $\psi$ to $\mathbb{R} / \mathbb{Z}$. Consider in $\mathbb{R} / \mathbb{Z} \times H$ the graph of $\psi$ (upside down). Let $L$ be its inverse image in $\mathbb{R} \times H$. On $G=\mathbb{R} \times H$, consider the automorphism $\tau_{\lambda}(x, h)=(\lambda x, h)$. 
Consider the path $\tau_{\lambda}(L)$ for $\left.\lambda \in\right] 0,+\infty[$. Clearly, when $\lambda \rightarrow 0$, it tends to $\mathbb{R} \times H$. When $\lambda$ tends to $+\infty$, we claim that it tends to $\{0\}$. Indeed consider $\left(\lambda_{i}\right) \rightarrow \infty$, and $\left(t_{i}, h_{i}\right) \rightarrow(t, h)$ with $\left(t_{i}, h_{i}\right) \in \tau_{\lambda_{i}}(L)$. This means that $\left(\lambda_{i}^{-1} t_{i}, h_{i}\right) \in L$. So $\psi\left(h_{i}\right)=\lambda_{i}^{-1} t_{i}$ in $\mathbb{R} / \mathbb{Z}$. As $\left(t_{i}, h_{i}\right) \rightarrow(t, h)$ and $\lambda_{i}^{-} 1 \rightarrow 0$, we have $\psi\left(h_{i}\right) \rightarrow$ $\psi(h)$ and $\lambda_{i}^{-1} t_{i} \rightarrow 0$. So $\psi(h)=0$ in $\mathbb{R} / \mathbb{Z}$. By injectivity of $\psi$, we deduce that $h=0$. We obtain that in restriction to the clopen subset $\mathbb{R} \times(H-\{0\})$, the path $\tau_{\lambda}(L)$ tends to $\varnothing$ when $\lambda$ tends to $+\infty$. In restriction to $\mathbb{R} \times\{0\}$, this is constantly $\{0\}$. So the claim is proved.

- $H^{\vee}$ is discrete and injects into $\mathbb{R} / \mathbb{Z}$. By Pontryagin-Chabauty duality, it amounts to join $\mathbb{R} \times\{0\}$ and $\mathbb{R} \times H^{\vee}$ in $\mathcal{S}\left(\mathbb{R} \times H^{\vee}\right)$. Using connectedness of $\mathcal{S}(\mathbb{R})$, we can join $\mathbb{R} \times\{0\}$ and $\{(0,0)\}$, respectively $\{0\} \times H^{\vee}$ and $\mathbb{R} \times H^{\vee}$, so it is enough to join $\{(0,0)\}$ and $\{0\} \times H^{\vee}$, which was done in the previous case.

Theorem 8.4 If $R(G) \geq 1$ then $\mathcal{S}(G)$ is connected. If moreover $G$ is metacircular (for example, if $G$ is a compactly generated Lie group), then $\mathcal{S}(G)$ is path-connected.

Proof First assume that $G$ is metacircular. Set $G=\mathbb{R}^{k} \times H$ with $k \geq 1$ and $R(H)=0$. In view of Proposition 8.2, it is enough to show that every closed subgroup of the form $\{0\} \times M$, can be joined to $\{0\}$ by a path. We can find a composition series

$$
\{0\}=M_{0} \leq \cdots \leq M_{n}=M
$$

such that each $M_{n} / M_{n+1}$ is either circular of the Pontryagin dual of a circular group. From Lemma 8.3 , we can join $\mathbb{R} \times M_{i}$ to $\mathbb{R} \times M_{i-1}$ by a path. Combining, we join $\mathbb{R} \times M$ and $\mathbb{R} \times\{0\}$. This proves the second statement.

The first statement is deduced by the projective limit argument. First assume that $G$ is a Lie group. Then $M$ is the direct limit of its open compactly generated subgroups $M_{i}$, so $\mathcal{S}(G)$ is the projective limit of $\mathcal{S}\left(\mathbb{R}^{k} \times G_{i}\right)$, which are compact and connected. So $\mathcal{S}(G)$ is connected.

By Pontryagin duality, we deduce that $\mathcal{S}(G)$ is connected when $G$ is any compactly generated LCA-group with $R(G) \geq 1$ (see Section 2.5). We can reiterate a second time the projective limit argument to deduce that $\mathcal{S}(G)$ is connected for any LCA-group with $R \geq 1$.

From Theorem 8.4, we see that $\mathcal{S}(G)$ can be path-connected even when $G$ is not a compactly generated Lie group, for instance when $G=\mathbb{R} \times D$ with $D$ a discrete group with a injection into $\mathbb{R} / \mathbb{Z}$. However, path-connectedness may fail for some groups with $R \geq 1$, as the following example shows. 
Proposition 8.5 Let $A$ be an infinite, discrete abelian group of uniform torsion. Then $\mathcal{S}(\mathbb{R} \times A)$ is not path-connected.

Proof Consider the natural map $\rho: \mathcal{S}(\mathbb{R} \times A) \rightarrow \mathcal{S}(\mathbb{R})\left(\rho=i_{\mathbb{R} \times\{0\}}\right.$ in the notation of Section 6). Identifying $\lambda \mathbb{Z}$ with $\lambda^{-1},\{0\}$ with 0 and $\mathbb{R}$ with $\infty$, we can view $\rho$ as a continuous map to $[0, \infty]$. We define maps $w, w^{\prime}: \mathcal{S}(\mathbb{R} \times A) \rightarrow \mathcal{S}(A)$ as follows: $w(H)$ is the projection of $H \subset \mathbb{R} \times A$ to $A$ and $w^{\prime}(H)$ is the intersection of $H$ with $\{0\} \times A$, identified with $A$.

In restriction to $\left.\left.\rho^{-1}(] 0,+\infty\right]\right)$, an easy compactness argument shows that the projection map $w$ is continuous. Besides, in restriction to $\rho^{-1}([0,+\infty[)$, the intersection map $w^{\prime}$ is continuous: indeed if $\left(H_{i}\right)$ tends to $H$, a priori $H_{i} \cap A$ could tend to something smaller than $H \cap A$. But if $(0, h) \in H \cap A$, it is approximable by elements $\left(e_{i}, h_{i}\right)$ of $H_{i}$; so $\left(d e_{i}, 0\right)$ belongs to $H_{i}$ (where $d A=0$ ); unless $e_{i}$ is eventually zero, this would imply that $\rho\left(H_{i}\right) \rightarrow \infty$, contradiction.

We claim that if $A_{0}$ and $A_{1}$ are subgroups of $A$, and if $\mathbb{R} \times A_{0}$ and $\mathbb{R} \times A_{1}$ can be joined by a path, then $A_{0}$ and $A_{1}$ are commensurable (the converse is an easy consequence of Theorem 8.4). Consider a path $\gamma$ in $\mathcal{S}(\mathbb{R} \times A)$ with $\gamma(i)=\mathbb{R} \times A_{i}$ $(i=0,1)$. By compactness, we can find $0=t_{0}<\cdots<t_{1}<\cdots<t_{k}=1$ such that each $\gamma\left(\left[t_{i}, t_{i+1}\right]\right)$ is contained in either $\left.\left.\rho^{-1}(] 0,+\infty\right]\right)$ or $\rho^{-1}([0,+\infty[)$. In the first case, $w\left(\gamma\left(t_{i}\right)\right)=w\left(\gamma\left(t_{i+1}\right)\right)$ by continuity and connectedness, and because $\mathcal{S}(A)$ is totally disconnected. Similarly, in the second case, $w^{\prime}\left(\gamma\left(t_{i}\right)\right)=w^{\prime}\left(\gamma\left(t_{i+1}\right)\right)$. But $w^{\prime}(H)$ is a finite index subgroup of $w(H)$ for any $H$, since any subquotient of $\mathbb{R}$ of uniform torsion is finite. Therefore in all cases, $w\left(\gamma\left(t_{i}\right)\right)$ is commensurable to $w\left(\gamma\left(t_{i+1}\right)\right)$. Accordingly $w\left(t_{0}\right)=A_{0}$ and $w\left(t_{k}\right)=A_{1}$ are commensurable.

We finish this section by a counterexample to Protasov and Tsybenko [26, Lemma 1], which at the same time answers positively a conjecture at the end at the same paper, namely that there exists a topological group $G$ with a closed subgroup isomorphic to $\mathbb{R}$, such that $\mathcal{S}(G)$ is not connected.

Proposition 8.6 Let $\Gamma$ be a non-solvable finite group. Then $\mathcal{S}(\mathbb{R} \times \Gamma)$ is not connected.

Proof If $\Lambda$ is a subgroup of $\Gamma$, the natural intersection map $i_{\Lambda}: \mathcal{S}(\Gamma) \rightarrow \mathcal{S}(\Lambda)$ is continuous and surjective, so replacing $\Gamma$ by a smaller subgroup if necessary (for example, the intersection of its derived series), it is enough to prove the proposition assuming that $\Gamma$ is perfect and non-trivial, that is, $[\Gamma, \Gamma]=\Gamma$. Then define $\mathcal{A} \subset$ $\mathcal{S}(\mathbb{R} \times \Gamma)$ as the set of subgroups containing $\{0\} \times \Gamma$. If $L$ is a subgroup of $\Gamma$, define $\mathcal{B}_{L} \subset \mathcal{S}(\mathbb{R} \times \Gamma)$ as the set of subgroups contained in $\mathbb{R} \times L$. These are clearly closed 
subsets. So the union $\mathcal{B}$ of $\mathcal{B}_{L}$, where $L$ ranges over proper subgroups of $\Gamma$, is closed. Clearly $\mathcal{B}$ consists whose subgroups whose projection to $\Gamma$ is a proper subgroup, and in particular $\mathcal{A} \cap \mathcal{B}=\varnothing$.

Now since $\Gamma$ is perfect, the derived subgroup of any element $H$ of $\mathcal{S}(\mathbb{R} \times \Gamma)-\mathcal{B}$ is exactly $\{0\} \times \Gamma$, so that $\mathcal{A} \cup \mathcal{B}=\mathcal{S}(\mathbb{R} \times \Gamma)$, which gives a partition into two closed subsets, which are both non-empty as soon as $\Gamma \neq\{1\}$.

\section{References}

[1] W Banaszczyk, New bounds in some transference theorems in the geometry of numbers, Math. Ann. 296 (1993) 625-635 MR1233487

[2] N Bourbaki, Éléments de mathématique. Fascicule XXIX. Livre VI: Intégration. Chapitre 7: Mesure de Haar. Chapitre 8: Convolution et représentations, Actualités Scientifiques et Industrielles 1306, Hermann, Paris (1963) MR0179291

[3] N Bourbaki, Éléments de mathématique. Fascicule XXXII. Théories spectrales. Chapitre I: Algébres normées. Chapitre II: Groupes localement compacts commutatifs, Actualités Scientifiques et Industrielles 1332, Hermann, Paris (1967) MR0213871

[4] N Bourbaki, Éléments de mathématique. Topologie générale. Chapitres 1 à 4, Hermann, Paris (1971) MR0358652

[5] D L Boyer, Enumeration theorems in infinite Abelian groups, Proc. Amer. Math. Soc. 7 (1956) 565-570 MR0078996

[6] M R Bridson, P de la Harpe, V Kleptsyn, The Chabauty space of closed subgroups of the three-dimensional Heisenberg group, Pacific J. Math. 240 (2009) 1-48 MR2485473

[7] C Chabauty, Limite d'ensembles et géométrie des nombres, Bull. Soc. Math. France 78 (1950) 143-151 MR0038983

[8] C Champetier, L'espace des groupes de type fini, Topology 39 (2000) 657-680 MR1760424

[9] Y de Cornulier, L Guyot, W Pitsch, On the isolated points in the space of groups, J. Algebra 307 (2007) 254-277 MR2278053

[10] Y de Cornulier, L Guyot, W Pitsch, The space of subgroups of an abelian group, J. Lond. Math. Soc. (2) 81 (2010) 727-746 MR2650794

[11] J Dixmier, Quelques propriétés des groupes abéliens localement compacts, Bull. Sci. Math. (2) 81 (1957) 38-48 MR0097457

[12] R Engelking, Dimension theory, North-Holland Mathematical Library 19, NorthHolland Publishing Co., Amsterdam (1978) MR0482697 Translated from the Polish and revised by the author

[13] S Fisher, P Gartside, On the space of subgroups of a compact group I, Topology Appl. 156 (2009) 862-871 MR2498918 
[14] S Fisher, P Gartside, On the space of subgroups of a compact group II, Topology Appl. 156 (2009) 855-861 MR2498917

[15] R I Grigorchuk, Degrees of growth of finitely generated groups and the theory of invariant means, Izv. Akad. Nauk SSSR Ser. Mat. 48 (1984) 939-985 MR764305

[16] T Haettel, L'espace des sous-groupes fermés de $\mathbb{R} \times \mathbb{Z}$, Algebr. Geom. Topol. 10 (2010) 1395-1415 MR2661531

[17] W Hurewicz, H Wallman, Dimension Theory, Princeton Mathematical Series 4, Princeton University Press, Princeton, NJ (1941) MR0006493

[18] J Isbell, Graduation and dimension in locales, from: "Aspects of topology", London Math. Soc. Lecture Note Ser. 93, Cambridge Univ. Press, Cambridge (1985) 195-210 MR787829

[19] Y Katuta, On the covering dimension of inverse limits, Proc. Amer. Math. Soc. 84 (1982) 588-592 MR643755

[20] B Kloeckner, The space of closed subgroups of $\mathbb{R}^{n}$ is stratified and simply connected, J. Topol. 2 (2009) 570-588 MR2546586

[21] K Mahler, Ein Übertragungsprinzip für konvexe Körper, Časopis Pěst. Mat. Fys. 68 (1939) 93-102 MR0001242

[22] L Narens, Topologies of closed subsets, Trans. Amer. Math. Soc. 174 (1972) 55-76 MR0312450

[23] B A Pasynkov, The coincidence of various definitions of dimensionality for locally bicompact groups, Dokl. Akad. Nauk SSSR 132 (1960) 1035-1037 MR0122906 Translated as Soviet Math. Dokl. 1 (1960) 720-722

[24] I Pourezza, J Hubbard, The space of closed subgroups of $\mathbb{R}^{2}$, Topology 18 (1979) 143-146 MR544155

[25] $\overline{\mathbf{I}}$ V Protasov, Dualisms of topological abelian groups, Ukrain. Mat. Zh. 31 (1979) 207-211, 224 MR530080

[26] $\bar{I}$ V Protasov, Y V Tsybenko, Connectedness in the space of subgroups, Ukrain. Mat. Zh. 35 (1983) 382-385 MR704344

[27] I V Protasov, Y V Tsybenko, Chabauty's topology in the lattice of closed subgroups, Ukrain. Mat. Zh. 36 (1984) 207-213 MR742446

[28] P Vopěnka, On the dimension of compact spaces, Czechoslovak Math. J. 8 (83) (1958) 319-327 MR0102068

[29] F Wattenberg, Topologies on the set of closed subsets, Pacific J. Math. 68 (1977) 537-551 MR0500894

Laboratoire de Mathématiques, Bâtiment 425, Université Paris-Sud 11

91405 Orsay, France

yves. cornulier@math.u-psud.fr

Received: 6 December $2010 \quad$ Revised: 8 April 2011 